CARNETS OE Carnets de géographes

GÉOGRAPHES

9| 2016

Géographies des émotions

\title{
Défendre et protéger son quartier de la
} destruction

Les émotions dans la mobilisation d'habitants contre les projets immobiliers dans le quartier Matta Sur, Santiago du Chili

\section{Clément Colin}

\section{(2) OpenEdition}

Journals

Édition électronique

URL : http://journals.openedition.org/cdg/555

DOI : $10.4000 /$ cdg. 555

ISSN : 2107-7266

Éditeur

UMR 245 - CESSMA

Référence électronique

Clément Colin, « Défendre et protéger son quartier de la destruction », Carnets de géographes [En ligne], 9 | 2016, mis en ligne le 30 novembre 2016, consulté le 19 avril 2019. URL : http://

journals.openedition.org/cdg/555; DOI : 10.4000/cdg.555

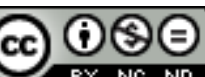

La revue Carnets de géographes est mise à disposition selon les termes de la Licence Creative Commons Attribution - Pas d'Utilisation Commerciale - Pas de Modification 4.0 International. 


\author{
DEFENDRE ET PROTEGER SON QUARTIER DE LA DESTRUCTION \\ Les émotions dans la mobilisation d'habitants contre les projets immobiliers dans le \\ quartier Matta Sur, Santiago du Chili
}

\author{
CLEMENT COLIN \\ Docteur en Géographie et Professeur associé \\ École de Travail Social \\ Pontificia Universidad Católica de Valparaíso \\ clement.colin@pucv.cl
}

\begin{abstract}
Résumé
Depuis les années 2000, des groupes d'habitants se mobilisent dans le centre de Santiago du Chili pour un double objectif. Le premier est de s'opposer à la destruction possible d'une partie de leur quartier à cause de la progressive multiplication de projets immobiliers dans ce secteur de la capitale. Le second objectif est la revalorisation patrimoniale et la protection du quartier. Comment s'organisent-ils et se structurent-ils ? Quels sens donnent-ils à leurs actions? A partir de ces questions, l'article propose une réflexion sur les fonctions des émotions ressenties et exprimées par les individus investis dans les mobilisations patrimoniales. La réflexion se fonde sur un travail de terrain effectué en 2014 et début 2015 auprès d'habitants du quartier Matta Sur, au centre de Santiago. Rencontres avec ces personnes, observations participantes lors de leurs actions et entretiens semi-directifs avec les principaux dirigeants des groupes servent à questionner ces processus socio-spatiaux.
\end{abstract}

Mots-clés : mobilisation patrimoniale, habitants, émotions, Santiago du Chili

\begin{abstract}
Since the 2000s, some groups of inhabitants are mobilized in the center of Santiago de Chile for a double objective. The first is the defense of their neighborhood from its potential destruction due to the rise of real estate projects in this sector of the city. The second objective is the heritage revalorization and the protection of the neighborhood. How are they structured and organized? What are the meanings that they give to their actions? From these questions, the paper proposes a reflection about the functions of the emotions felt and expressed by the participants of these heritage mobilizations. The discussion is based on an ethnographic field work conducted in 2014 and 2015 with residents of the Matta Sur neighborhood, in the center of Santiago. Participating observations, meetings with inhabitants and semi-structured interviews with the leaders of the mobilization movement are used in order to analyze these social-spatial processes.
\end{abstract}


Carnets de géographes, no 9, Septembre 2016

Rubrique Carnets de recherches

Keywords: heritage mobilization, inhabitants, emotions, Santiago du Chili 


\section{Note de l'auteur}

L'auteur remercie le soutien du projet CEDEUS CONICYT/FONDAP n¹5110020.

\section{Introduction}

Depuis les années 2000, des groupes d'habitants se mobilisent dans le centre de Santiago du Chili pour un double objectif. Le premier est de s'opposer à la possible destruction de leur quartier due à la multiplication de projets immobiliers dans ce secteur de la capitale depuis les années 1990. Ils veulent ainsi faire face à un processus de densification du centre impulsé par la municipalité et l'Etat durant ces années (Hidalgo, 2010). Le second objectif de ces rassemblements est la protection et la revalorisation patrimoniale du quartier. Pour cela, ils manifestent dans les rues principales de la ville, ils organisent des réunions publiques, ils demandent plus de protection au Conseil des Monuments Nationaux et à la municipalité et ils diffusent leurs messages sur Internet. Par leurs actions, ces groupes transforment progressivement leur quartier en lieu-support de revendications sociales et parfois politiques. D'une protestation contre la dégradation et le risque de destruction de quartiers au centre de Santiago, cette mobilisation est aujourd'hui devenue un mouvement à dimension nationale présent dans de nombreux quartiers de la capitale et dans plusieurs villes au Chili (Canteros, 2011). Dispersés en leurs débuts, les groupes d'habitants sont depuis 2009 coordonnées à travers l'Asociación chilena de los barrios y zonas patrimoniales, fondée par des habitants du quartier Yungay, au centre de Santiago, à la suite de leur victoire sur les promoteurs immobiliers qui s'est traduite par le classement d'une partie de ce secteur. Grâce à ces évolutions, aujourd'hui, une réglementation plus stricte est en vigueur dans les plans d'urbanisme.

L'article interroge la mobilisation d'habitants pour la revalorisation patrimoniale de leur quartier à travers les émotions exprimées et partagées par les participants. En quoi ces émotions organisent-elles les interrelations individuelles au sein du groupe et conduisentelles à la mobilisation ? Plus précisément, en quoi la mobilisation d'habitants révèle-t-elle des groupes formés sur une base émotionnelle partagée dont l'espace serait à la fois la source et le support? Le travail se fonde sur l'hypothèse que nous serions face à des groupes formés à partir d'une double dynamique émotionnelle : la première, structurante, est engendrée par leur attachement au quartier et la seconde, conductrice de la mobilisation, se construit en lien avec le cadre politique et économique actuel. D'un côté, ce type de groupe serait composé d'habitants qui interagiraient et s'organiseraient collectivement à partir d'émotions communes ressenties vis-à-vis de leur espace de vie : le quartier. D'un autre côté, le risque de disparition ou de transformation du même espace engendrerait d'autres types d'émotions qui conduiraient les habitants à s'organiser autour d'actions, de pratiques collectives et à diffuser des discours revalorisant le quartier.

L'article se fonde sur un travail ethnographique réalisé en 2014 et au premier semestre 2015 avec les habitants engagés dans des groupes dont l'objectif est la défense et la revalorisation patrimoniale d'un quartier de Santiago Centre: Matta Sur. Nous nous intéressons principalement à deux groupes : le Centro cultural Patrimonio Matta Sur fondé en 2009 et le Comité de defensa del barrio Matta Sur créé en 2007. Pour le premier, nous avons assisté à six réunions organisées pour la création d'un musée du quartier entre mai 2014 et mars 2015 et d'avril à mai de la même année, nous avons aidé à l'élaboration d'un registre de 
photographies patrimoniales du même quartier dans le cadre d'ateliers de deux à trois heures deux fois par semaine (financés grâce à l'obtention d'un fonds sur concours du ministère de la Culture). Pour le second groupe, nous avons participé à différentes de leurs actions, comme par exemple la plantation de fleurs ou d'arbustes dans les plates-bandes dans les rues du quartier en mars 2014 ou le défilé " Marcha por el patrimonio " organisé par l'Asociación chilena de los barrios y zonas patrimoniales en mai 2014. Nous avons aussi rencontré les responsables de ce groupe dans différentes réunions organisées par le premier. A travers notre participation, nous avons discuté de manière régulière avec les participants et nous avons ainsi pu mieux comprendre leurs motivations, leurs attentes et leurs intérêts. En plus de l'analyse des textes des documents diffusés par les associations d'habitants (bulletins, page Facebook, sites Internet) et afin de compléter les éléments ethnographiques, nous avons aussi mené des entretiens semi-directifs avec quatre des dirigeants d'associations du quartier.

L'argumentation s'organise en six parties. La première s'intéresse au cadre théorique et méthodologique à l'intérieur duquel s'inscrit l'étude. Nous focalisons ainsi notre réflexion sur les liens entre émotions et mobilisation patrimoniale. La seconde partie aborde la contextualisation de l'étude de cas afin de mieux comprendre ses dimensions sociales et politiques. Nous interrogeons ainsi l'origine des revendications des habitants mobilisés dans les quartiers centraux de Santiago et nous présentons notre cadre d'analyse : le quartier Matta Sur. La troisième partie porte sur les groupes étudiés, leurs projets et leurs objectifs. La quatrième partie s'intéresse à l'attachement affectif au quartier exprimé par les participants en tant que base de la mobilisation. Dans la cinquième partie, nous interrogeons la manière dont les émotions individuelles se transforment en émotions collectives supports et ressources de la mobilisation. Enfin, la sixième partie questionne les émotions comme normes qui structurent et délimitent les groupes.

\section{Les émotions et leurs fonctions dans la mobilisation patrimoniale}

\section{L'étude des émotions dans la mobilisation sociale}

Longtemps étudiées sous l'angle des modèles de l'action rationnelle (Olson, 1992), de la mobilisation de ressources (Tilly, 1978; McCarthy, Zald, 1977) et de la structure des opportunités politiques (McAdam, 1999; Tarrow, 1989), les mobilisations sociales ne peuvent se résumer à l'accumulation de calculs stratégiques et rationalistes acceptés par un ensemble d'individus avec des intentions communes et précises a priori. Les participants évaluent les objectifs de la mobilisation selon leurs pensées, leurs histoires, leurs interprétations propres associées à leur milieu social mais aussi basées sur leurs expériences quotidiennes (Schütz, 1998). Ils ne donnent ainsi parfois le même sens ni à leurs actions ni à leurs finalités. De plus, une personne ne choisit pas d'agir à partir d'un seul type de rationalité mais plutôt de nombreux registres d'engagements qui s'articulent, se complètent et parfois se contredisent (Boltanski, Thévenot, 1991). Les mobilisations sociales peuvent ainsi être abordées comme le résultat d'un processus à l'intérieur duquel des individusacteurs prennent des décisions, négocient et parfois entrent en conflit à propos des causes et des objectifs qui guident la protestation. Ces rapports entre individus servent aussi à l'élaboration d'une identité collective (Melucci, 1999) avec des orientations, des objectifs, des pratiques, des discours et des intérêts communs. 
Par ailleurs, se mobiliser et participer à des actions collectives peuvent être aussi vus comme une expérience partagée avec d'autres participants. Dans ce cadre, les émotions jouent un rôle à la fois dans le choix de se joindre à la cause du groupe (Traïni, 2009) et dans les modes d'interactions entre acteurs (Goodwin et al., 2001). Elles sont en effet présentes dans toutes les phases et les aspects des protestations sociales (Jasper, 2012) : I'honneur, la fierté, la vengeance ou la reconnaissance sociale et morale d'un groupe sont souvent au cœur de sa formation. Cependant, elles sont souvent complexes et évolutives (Collins, 2001). Une personne peut expérimenter différentes émotions un même jour. De même, elle peut se mobiliser contre une situation qu'elle juge injuste, avoir de la colère, du mépris et même de la haine et du dégoût pour les personnes qu'elle juge responsables, mais en même temps, elle peut sentir de l'affection pour son groupe de lutte, émotions que transforment sa participation en plaisir indépendamment des objectifs du mouvement. Ces différents aspects jouent un rôle dans les choix de participation, d'action et de ralliement à la mobilisation.

Enfin, les émotions peuvent être utilisées afin de rallier de nouvelles personnes à la cause mais aussi à des fins personnelles dans le cadre d'ambitions politiques. Pour Christophe Traïni (2015), la coordination d'actions collectives s'appuie non seulement sur des dispositifs d'experts mais aussi sur des " dispositifs de sensibilisation " qu'il définit comme "l'ensemble des supports matériels, des agencements d'objets, des mises en scènes que les acteurs déploient afin de susciter des réactions affectives qui prédisposent ceux qui les éprouvent à soutenir la cause " (2015: 19). Dans cette optique, les mobilisations sociales peuvent être abordées comme un moyen pour médiatiser des émotions qui pourraient être partagées par d'autres (Flam, King, 2005). Par l'expression de leurs émotions dans l'espace public et par la diffusion de leurs discours, les participants peuvent "toucher " d'autres personnes qui seraient tenté d'appuyer ou de défendre la cause du groupe.

\section{La mobilisation patrimoniale comme phénomène émotionnel}

Le patrimoine est compris dans cet article comme le résultat d'une construction sociale (Prats, 2009). Il est interrogé en tant que produit d'actions, d'usages et de discours portés par des individus, des groupes ou des institutions qui réinterprètent les sens et les significations de l'objet potentiellement patrimoine en fonction de leurs intérêts, de leurs connaissances et de leurs attentes. Néanmoins, tout comme d'autres auteurs (Heinich, 2012 ; Fabre, 201“), nous pensons la patrimonialisation comme un phénomène d'abord émotionnel transformé a posteriori en processus social, politique et parfois économique basé sur des valeurs partagées ou imposées après négociations entre les acteurs concernés. Ce sont les liens affectifs que des personnes peuvent avoir pour un objet ou un espace qui les conduisent à vouloir le défendre, le protéger ou le revaloriser comme patrimoine. La patrimonialisation révèle ainsi non seulement un système de valeurs et de significations socio-culturelles que lui associent des individus mais aussi les relations affectives qu'entretiennent ces mêmes individus avec l'objet patrimonial en question.

Autre dimension essentielle pour comprendre la patrimonialisation : un patrimoine est le résultat d'une reconstruction mémorielle d'un passé dans le présent (Harvey, 2001 ; Smith, 2006) effectuée à partir de savoirs et de connaissances scientifiques ou amateurs mais aussi à partir des souvenirs de ses défenseurs. Nous retrouvons ainsi dans la mobilisation patrimoniale les dispositifs de coordination identifiés par Traïni (2015) : d'un côté les dispositifs de sensibilisation qui s'appuient sur les souvenirs, les mémoires de ses participants et de l'autre les dispositifs experts qui se fondent sur les travaux d'historiens, 
d'architectes ou de consultants auprès des institutions. D'un côté un savoir issu de la quotidienneté des personnes concernées par l'objet potentiellement patrimoine et de l'autre une expertise normative et institutionnelle basée sur la connaissance de spécialistes s'opposent et se contredisent parfois dans le cadre du processus de patrimonialisation.

La mobilisation patrimoniale est donc une mobilisation sociale avec un objectif précis : la protection, la conservation et la revalorisation patrimoniales. Il s'agit d'un processus social mené par un groupe d'individus dans l'objectif de promouvoir une idée de la revalorisation de l'espace urbain qui ne se ferait pas à travers les projets immobiliers mais plutôt par la conservation de son patrimoine à la fois matériel et immatériel et où les habitants auraient toute leur place dans les prises de décisions.

\section{Aborder les fonctions des émotions dans la mobilisation patrimoniale}

Pour définir les émotions, nous nous appuyons d'abord sur les travaux de Damasio. II propose de "réserver le terme de sentiment à l'expérience mentale et privée d'une émotion, et d'utiliser au contraire, le terme d'émotion pour désigner l'ensemble des réponses, qui pour bon nombre d'entre elles sont publiquement observables " (Damasio, 1999 : 50). Cependant, les émotions répondent aussi à des normes sociales et à des modèles culturels historiquement situés. Partant du même constat, Le Breton (2004) explique qu'elles naissent d'une relation à un objet et de l'évaluation qu'un individu fait d'une situation dans laquelle il est engagé. Evaluation qui se fait en fonction des valeurs socio-culturelles qu'il partage avec son groupe d'appartenance et plus généralement avec la société dans laquelle il vit. L'auteur questionne les émotions et ses expressions corporelles en tant que forme de communication entre individus qui partagent un même "répertoire culturel ». Ainsi, les expressions émotionnelles ont des significations socio-culturelles interprétées par les autres individus dans divers contextes de la vie quotidienne (Lutz, 1986).

Dans cet article, nous interrogeons les émotions exprimées publiquement (consciemment ou inconsciemment) par les participants afin de mieux comprendre leurs motivations et les significations qu'ils associent à leurs actions dans l'espace. Aborder sous cet angle les mobilisations conduit aussi à une meilleure compréhension des liens qui unissent les habitants à leur quartier et des valeurs qu'ils lui leur associent.

\section{Matta Sur, un quartier relativement épargné par les projets immobiliers}

\section{Les origines et les conséquences du développement urbain à Santiago du Chili}

La spéculation immobilière qui s'observe actuellement dans le centre de Santiago est le résultat de plans concertés entre les investisseurs privés et les autorités locales (Hidalgo, 2010) dans l'objectif de densifier le centre-ville et d'inverser la tendance à sa désertification et à la périurbanisation. Tandis qu'en 1940 la commune de Santiago Centre représentait 440000 habitants, ils n'étaient plus que 298877 dans les années 1970 (Contreras, 2011). Une des premières initiatives dans ce sens a été la libéralisation du marché du sol à partir de la «politique nationale de développement urbain » décidée en 1979. Une autre origine du développement immobilier est la création de la Corporation du développement de Santiago (Cordesan) - une institution privée dépendante de la municipalité - dont l'objectif est d'accompagner le repeuplement du centre. A partir de 1992, le Cordesan met en place des " plans de repeuplement " pour la commune de Santiago basés sur la délimitation de 
"zones de rénovation", la création d'une aide spécifique pour l'achat de logements et la dérégulation des normes de constructions établies par le plan régulateur de 1939. A cela s'ajoute un effort de la municipalité pour changer l'image du centre en améliorant les espaces publics et les passages piétons (par un meilleur éclairage nocturne par exemple) afin d'attirer de nouveaux habitants et investisseurs dans le secteur.

Figure 1 : Nouvelles constructions, Avenue Matta, Santiago Centre

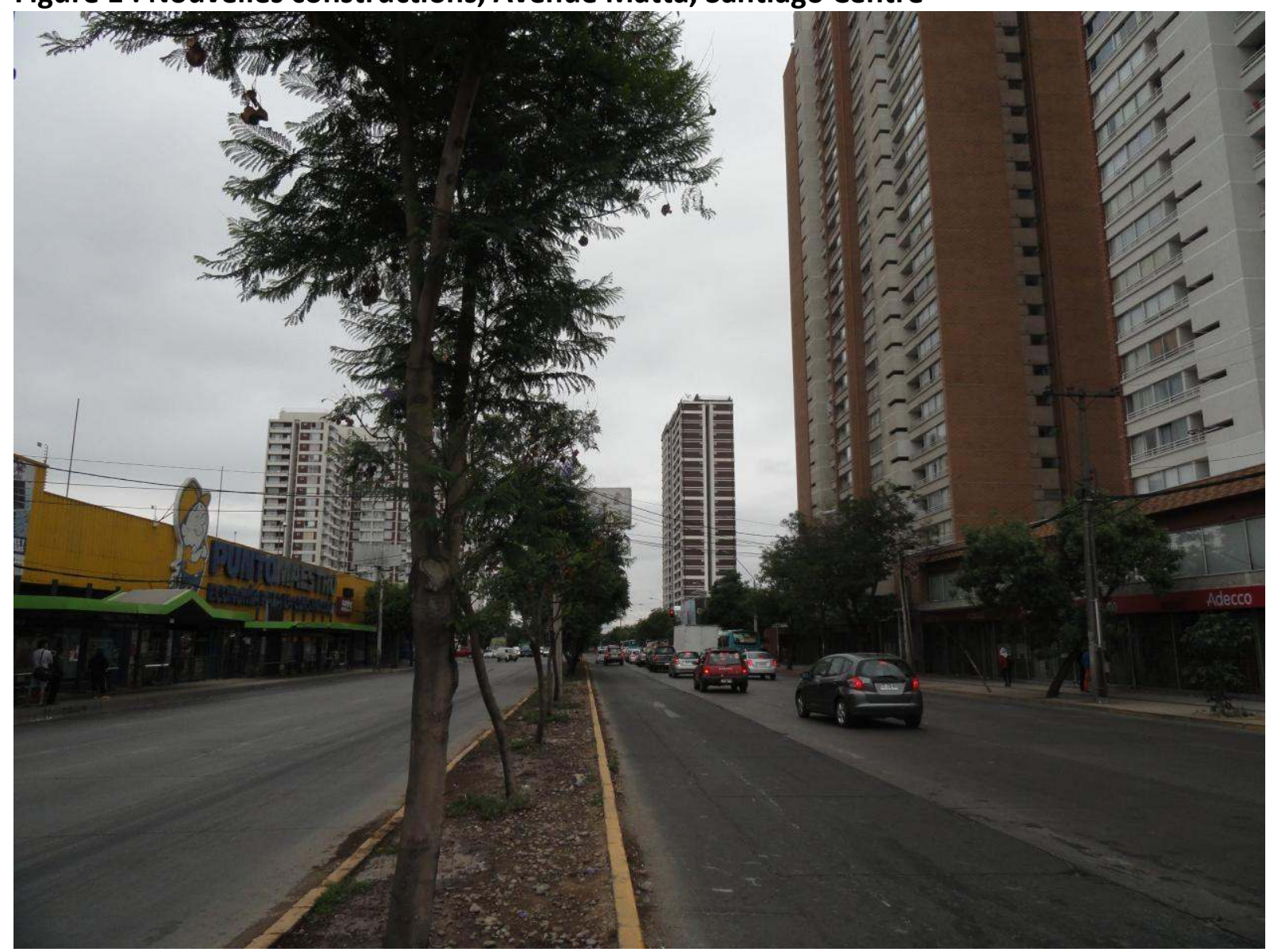

Cliché de l'auteur, 2015.

Une des conséquences les plus visibles de cette politique est aujourd'hui la verticalisation du centre de Santiago. Depuis leurs débuts, les plans de repeuplement ont permis la construction de plus de 100000 logements dans plus de 500 opérations immobilières (Contreras, 2011), détruisant ainsi des secteurs entiers du centre en remplaçant les maisons ou les immeubles anciens par des tours dépassant parfois plus de trente étages (Figure 1). Une autre conséquence de ces investissements immobiliers est sociale: l'arrivée de nouveaux habitants conduit à un renouvellement de la population qui s'accompagne d'un changement de son profil socio-économique. La majorité d'entre eux sont des familles monoparentales, des célibataires ou des couples sans enfant qui choisissent de venir vivre dans le centre pour les commodités que cela peut apporter en termes d'accessibilité et de services, pour la proximité de leurs réseaux sociaux, professionnels ou familiaux mais aussi pour les valeurs sociales qu'ils donnent au fait d'habiter au centre (Contreras, 2011). Ils se caractérisent aussi par leur multi-appartenance sociale et spatiale permise par leur grande mobilité à différents échelles territoriales (du quartier à l'agglomération). Cependant, il ne s'agit souvent pas de gentrification, dans le sens où ils n'ont en général pas de revenus 
supérieurs aux habitants déjà présents dans ces quartiers (Hidalgo, 2010). Finalement, les nouveaux résidents se composent aussi des immigrants en majorité péruviens (Torres et Hidalgo, 2009) ou colombiens, attirés par l'offre d'appartements de petite taille dans les tours ou dans les anciennes maisons en mauvais état.

\section{Le quartier Matta Sur, un quartier résidentiel au cœur de l'agglomération}

Afin d'interroger les émotions présentes dans la mobilisation patrimoniale, nous nous intéressons à deux groupes situés dans un même quartier: Matta Sur, au centre de l'agglomération de Santiago du Chili (Figure 2).

Figure 2 : Localisation du quartier Matta Sur dans l'agglomération de Santiago du Chili

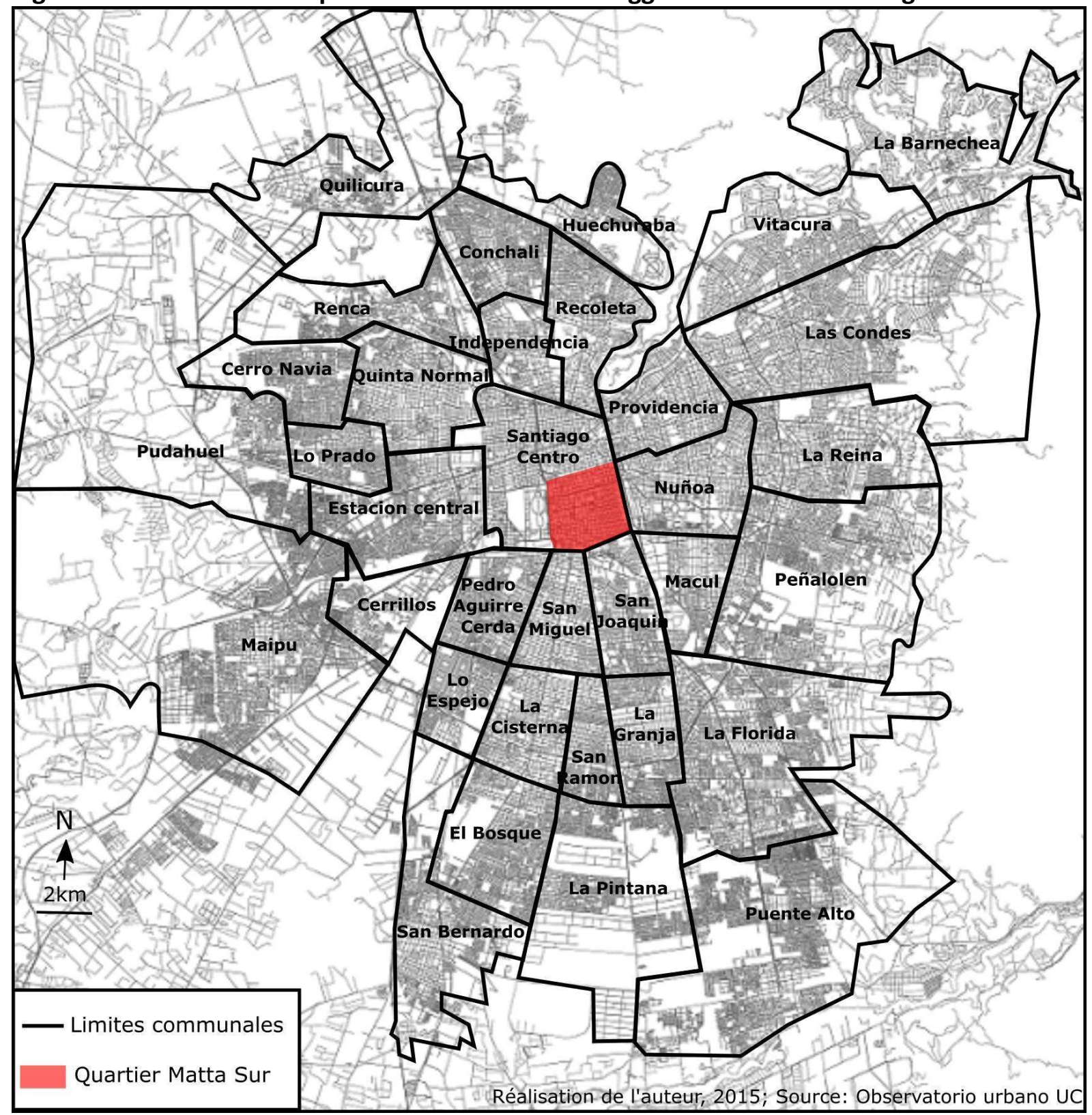

Avant le XIX ${ }^{\mathrm{e}}$ siècle, le quartier Matta Sur se compose de fermes et de petits hameaux constitués autour de chemins et de rues boueuses. A partir du XIX ${ }^{\mathrm{e}}$ siècle, les habitats précaires de cette zone se multiplient à cause d'une importante migration rurale, formant 
ainsi une vaste zone urbaine précaire connue pour ses conditions de vie très difficiles (Ramón, 2000). A partir de 1875 et tout au long du XX ${ }^{\mathrm{e}}$ siècle, cet espace autrefois rural se transforme en un secteur urbain et industriel. Matta Sur est ainsi bâti progressivement par la vente d'îlots où se construisent des poblaciones, c'est-à-dire des habitats ouvriers devant répondre aux besoins de logements dû à l'exode rural très important à cette époque.

\section{Figure 3 : Les différents secteurs du quartier Matta Sur}

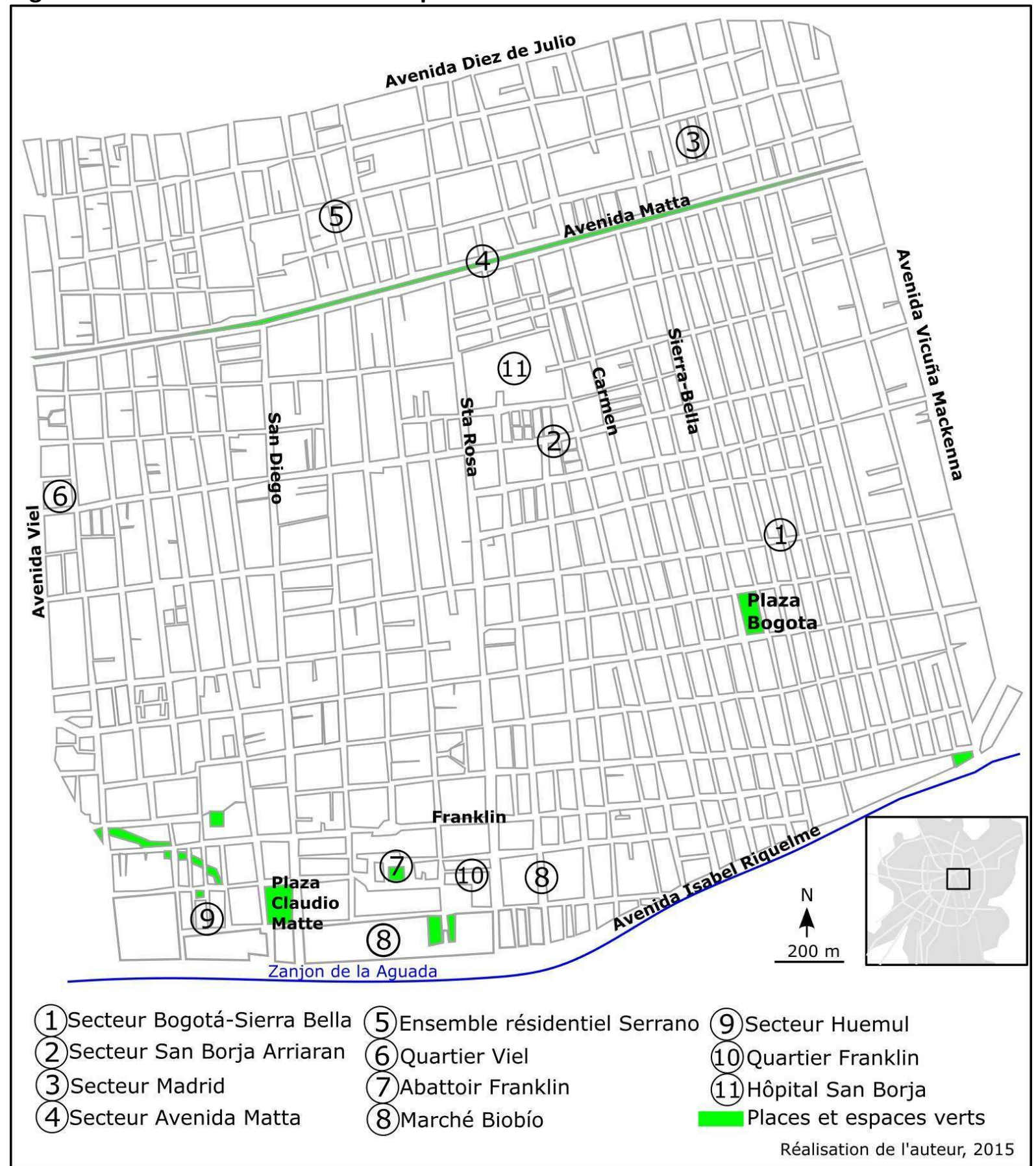

Au recensement de 2002 (dernier en date), ce secteur représentait 51217 habitants, soit 25,5\% de la population de Santiago Centre (Ilustre Municipalidad de Santiago, 2013). II est composé aujourd'hui en majorité par des habitants de classe moyenne inférieure et seul $1 \%$ de la population peut être considéré comme de classe supérieure (Ilustre Municipalidad de 
Santiago, 2013). Les familles les plus " aisées » habitent dans le secteur de la place Bogotá, au centre-est du quartier. Dans le secteur sud nommé "Franklin » (Figure 3), les habitants appartiennent à des catégories socio-économiques moins élevées que dans le reste du quartier. Sur le plan des activités économiques, Matta Sur se compose d'ateliers, de petites industries et de petits commerces. La conversion de nombreuses maisons en ateliers (notamment pour la réparation de voitures) ou en entreprises de service conduit même certains habitants à qualifier Matta Sur de quartier industriel. Néanmoins, lors de la modification du plan régulateur du secteur en 2013, la municipalité fait le constat que $22 \%$ des propriétés correspond à des activités productrices, $19 \%$ à des équipements municipaux ou privés et $53,7 \%$ à de l'usage résidentiel. Sur un plan urbanistique, cet espace urbain est composé en majorité d'édifices en rez-de-chaussée (70,9\%) et d'un étage (24\%) (Ilustre municipalidad de Santiago, 2013). De plus, la majorité des constructions (70,9\%) datent d'avant 1950 et seuls 2,8\% des édifices ont été construits entre 2001 et 2007 (llustre municipalidad de Santiago, 2013). Le quartier Matta Sur est donc relativement préservé des constructions élevées pour le moment mais la menace est toujours persistante (Figure 4). Pour les habitants de Matta Sur, les tours sont le symbole de la modernisation destructrice enclenchée par la municipalité et conduite par les promoteurs immobiliers. Ils les redoutent et en ont peur car elles sont synonymes de changements à la fois sociaux, économiques et culturels dans le quartier.

Figure 4 : Vue d'un immeuble en construction depuis une rue du secteur Bogota

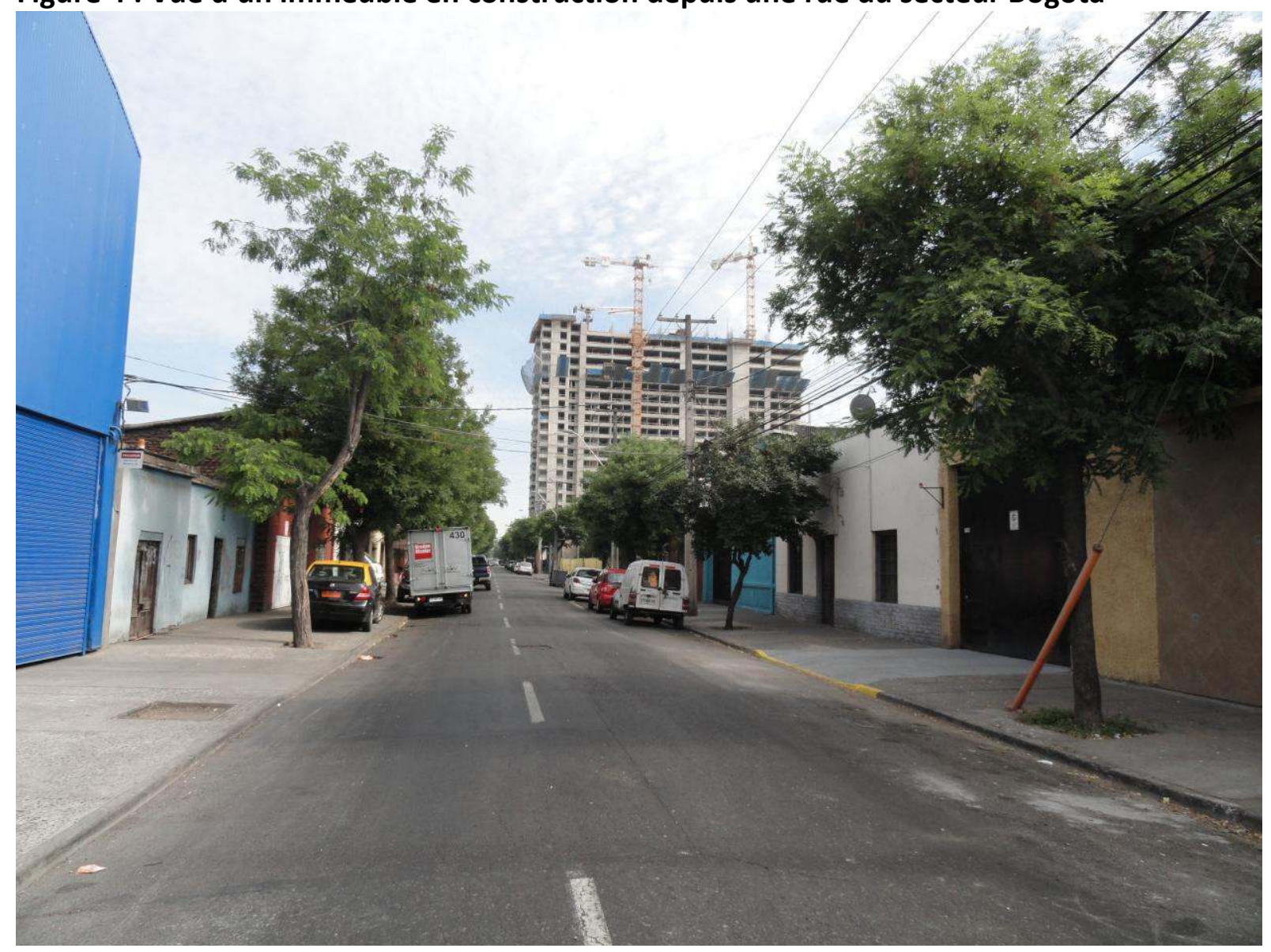

Cliché de l'auteur, 2015. 
En décembre 2015, après huit années de négociations entre les groupes d'habitants et le Conseil des Monuments Nationaux et grâce à l'appui politique de la municipalité à partir de 2013, une grande partie de Matta Sur est déclarée zona típica, c'est-à-dire zones de protection patrimoniale ${ }^{1}$. Notre analyse porte sur la période de mobilisation que précède ce classement. Jusqu'à décembre 2015, le processus de patrimonialisation était conduit surtout par ses habitants. Les institutions n'intervenaient qu'indirectement à travers le financement de quelques-uns des projets mis en place par ces groupes (Figure 5). Jusqu'à la délimitation de la zone de protection, il s'agissait donc d'un désir de patrimonialisation fondé sur la quotidienneté et les souvenirs et mémoires familiales des habitants. Un processus où les émotions jouent un rôle prépondérant.

\section{Présentation des deux groupes étudiés}

Le Comité de defensa del barrio Matta Sur et le Centro cultural Patrimonio Matta Sur font partie d'une constellation beaucoup plus grande de groupes d'habitants qui se retrouvent dans différents quartiers et communes de Santiago et de différentes villes au Chili. En s'organisant collectivement contre les interventions urbaines sur leur territoire, ils cherchent à s'imposer comme nouvel acteur urbain (Sabatini et Wormald, 2004 ; Canteros, 2011). Parfois officialisés sous le statut d'association ou de centre culturel, parfois non reconnus juridiquement, leurs moyens d'actions ne sont pas équivalents selon les territoires et les profils socio-économiques des participants (Poduje, 2008).

Le Comité Matta Sur est fondé en 2007. L'objectif central était de s'opposer au projet de bus en site propre qui occuperait le centre l'Avenue Matta et détruirait les espaces verts existants. Après leur victoire contre le ministère des transports, le Comité a étendu ses revendications à la protection du quartier Matta Sur et notamment à la délimitation d'une zona típica comprenant l'ensemble urbain visible sur la Figure 3. L'autre groupe - le Centro cultural Patrimonio Matta Sur - est né en 2009 du premier avec comme objectif la revalorisation patrimoniale du quartier. Les deux groupes sont en grande partie financés par des fonds sur concours provenant de la municipalité, de la région métropolitaine ou de l'Etat. Ils représentent chacun entre trente à quarante ${ }^{2}$ personnes qui vivent pour la grande majorité dans le secteur Bogota-Sierra Bella (Figure 3) et qui sont pour la plupart inscrits dans les deux groupes à la fois. Ils sont des " établis " (Elias, Scotson, 1997), c'est-à-dire des individus qui sont nés en majorité dans le quartier et qui vivent parfois depuis toujours dans la même maison dont ils sont aujourd'hui propriétaires. Ils sont composés d'une majorité de femmes au foyer et de retraités (hommes et femmes). Il est aussi possible de rencontrer de jeunes actifs sensibles aux projets proposés pour le quartier. Dans l'ensemble, nous observons que les habitants mobilisés cherchent dans un premier temps à protéger leurs biens privés. Ce n'est que dans un second temps, influencés par les discours des dirigeants et par leurs échanges avec les autres participants, qu'ils prennent conscience de la dimension collective de leur lutte.

\footnotetext{
${ }^{1}$ Au Chili, il existe deux grands types de zonage patrimonial : le sector de conservación histórica, décidé par la municipalité, qui a un caractère plus indicatif que prescriptif et la zona típica, déclarée par le Conseil des Monuments Nationaux, qui s'accompagne de règles prescriptives.

${ }^{2}$ II n'existe pas de registre officiel et l'inscription est gratuite.
} 
Ces groupes sont conduits par des personnes aux capitaux social et culturel forts, capables d'adapter leurs discours aussi bien pour les habitants que pour les autorités publiques. Les dirigeants travaillent comme professeurs, scientifiques ou dans des institutions culturelles comme des musées. Ils organisent, coordonnent et mènent des projets à destination des habitants du quartier et de Santiago (Figure 5). Leur formation professionnelle et leurs expériences dans les milieux associatifs leur permettent de formuler des demandes de subventions et de transmettre les documents requis pour la demande de protection du quartier. Pour le Comité Matta Sur, le président travaille à temps complet dans les activités de l'association et sa femme, professeure des écoles, est chargée de la communication. Ils sont aidés par d'autres habitants dont la plupart sont retraités, mais actifs dans les milieux associatifs depuis de nombreuses années à Santiago. Afin d'atteindre leurs objectifs, ils mobilisent leurs réseaux qui se composent de personnalités des milieux associatifs et politiques de Santiago. Pour le Centro cultural Patrimonio Matta Sur, le président travaille pour un musée à Santiago où il organise des expositions. II n'est pas originaire du quartier mais il a vécu dans des secteurs aux caractéristiques similaires, ce qui lui donne une certaine légitimité auprès des autres habitants. D'une cinquantaine d'années, il est passionné par l'histoire sociale de son quartier. Par les activités qu'il propose aux résidents, il veut leur montrer qu'ils peuvent être fiers de leur héritage, de leur mode de vie : il cherche à leur redonner une certaine estime de soi. Il est aidé par un autre résident avec qui il a tissé des liens d'amitié. Ce dernier est professeur dans le secondaire et est originaire du quartier. II partage les mêmes ambitions que le président du groupe. Ils sont tous les deux connus des institutions car ils fréquentent les nombreuses réunions et séminaires organisés par ces dernières. Tout comme les dirigeants du Comité Matta Sur, ils sont très souvent sollicités par les médias pour traiter de sujets liés au patrimoine.

Les dirigeants des deux groupes étudiés sont appréciés par les autres participants. Lors des réunions, ils sont écoutés avec attention et ils sont généralement très applaudis. Le président du Centro cultural est connu dans le quartier car il va beaucoup à la rencontre des habitants lors des marchés ou des routes patrimoniales qu'il guide certains week-ends. II lui arrive aussi souvent d'inviter les participants (et même ses voisins non mobilisés) à venir prendre un café ou un repas chez lui. Son collègue est aussi très apprécié. Lors des ateliers de photographies, il était possible d'observer une certaine affinité entre lui et les participants qui se révélaient par des blagues et des rires avant qu'il ne prenne la parole. La bonne entente et la bonne humeur sont des bases qui permettent à ce groupe d'attirer de nouvelles personnes et de maintenir ses activités sur le long terme. 
Figure 5 : Quelques grands projets menés par les deux groupes

\begin{tabular}{|c|c|c|}
\hline Année & Comité Matta Sur & Centro cultural Patrimonio Matta Sur \\
\hline $2007-2015$ & \multicolumn{2}{|c|}{ Demande de la délimitation d'une zona típica pour l'ensemble du quartier Matta Sur } \\
\hline $\begin{array}{l}2013- \\
\text { aujourd'hui }\end{array}$ & \multicolumn{2}{|c|}{$\begin{array}{l}\text { Soutien politique et institutionnel de la maire* de Santiago Centre pour la demande de } \\
\text { protection et pour les actions et projets des deux groupes }\end{array}$} \\
\hline 2014 & $\begin{array}{l}\text { Coordination d'un projet de création de } \\
\text { jardins dans les platebandes des rues du } \\
\text { quartier }\end{array}$ & \\
\hline 2015 & $\begin{array}{l}\text { Campagne de sensibilisation avec la } \\
\text { municipalité sur le thème des ordures } \\
\text { nommé « No Mates Matta } \text { (Ne tues pas } \\
\text { Matta)** }\end{array}$ & \\
\hline 2014-2015 & & $\begin{array}{l}\text { Mise en place d'un musée du quartier itinérant, } \\
\text { nommé \& muestra en viaje "s, à partir de la } \\
\text { participation des habitants }\end{array}$ \\
\hline 2015 & & $\begin{array}{l}\text { Elaboration d'un registre de photographies } \\
\text { patrimoniales durant les mois d'avril, mai et juin où } \\
\text { ont participé quarante habitants du quartier. }\end{array}$ \\
\hline 2015 & & $\begin{array}{l}\text { Expositions des résultats des deux activités dans } \\
\text { différents secteurs du quartier et de Santiago } \\
\text { (Ecolespubliques, hôpitaux, durant lesmarchés) }\end{array}$ \\
\hline $\begin{array}{l}\text { Décembre } \\
2015\end{array}$ & & $\begin{array}{l}\text { Exposition dans le hall de la mairie de Santiago } \\
\text { Centre }\end{array}$ \\
\hline $\begin{array}{l}\text { Décembre } \\
2015\end{array}$ & Quartier Matta Sur déclaré Zona típ & ica par le Conseil des Monuments Nationaux \\
\hline
\end{tabular}

- Carolina Toha, Partido por la Democracia (PPD), parti de gauche, est maire de la commune de Santiago Centre depuis 2012.

*- Le problème des traitements des ordures est une problématique récurrente à Santiago (Sabatini et Wormald, 2004). Pour le cas de Matta Sur, il s'agit d'un point de conflit important entre les habitants et les propriétaires des entreprises.

Elaboration de l'auteur, 2015.

Parallèlement à ces activités et projets, les deux groupes sont présents lors des marchés et événements culturels dans le quartier. Ils participent aussi aux activités de l'Asociación chilena de los barrios y zonas patrimoniales telles que la "Marcha por el Patrimonio » en mai 2014 et 2015 ou les Congrès de l'association qui se déroulent une fois par an dans différentes villes au Chili. Enfin, ils font connaitre leurs idées et leurs actions à travers Internet et par leur bulletin nommé « El Adoquin », financé par la municipalité.

Leurs liens étroits avec le milieu politique et leur capacité à être présents sur les réseaux sociaux facilitent le ralliement d'habitants d'autres quartiers de Santiago à leur cause. De plus, ces deux groupes peuvent compter sur l'appui des autres associations défendant les mêmes objectifs à Santiago. Ils profitent aussi du soutien d'étudiants et d'universitaires travaillant sur ces thématiques et le plus souvent issus des facultés d'architecture ou de sciences sociales. Finalement, nous pouvons dire que ces mobilisations s'inscrivent dans un contexte social favorable à leurs revendications. La société chilienne est de plus en plus réceptive aux questions patrimoniales et environnementales. Les contestations sociales face aux projets d'équipement ou d'aménagement du territoire ne cessent de se développer depuis les années 2000 que ce soient contre les autoroutes à Santiago, contre les barrages hydroélectriques au sud du Chili ou contre la construction de centres commerciaux sur des sites historiques. La lutte contre les projets immobiliers à Santiago Centre peut ainsi compter sur le soutien ou du moins la sympathie d'une partie de la population et de certains responsables politiques. 


\section{L’attachement affectif au quartier transformé en référence commune aux groupes}

Les groupes étudiés se structurent à partir de relations affectives mutuelles pour leur quartier et certains de ses habitants. Cela se traduit généralement dans leur discours par de la nostalgie engendrée par les souvenirs du quartier "avant". La défense du quartier s'apparente alors souvent à une lutte pour préserver les souvenirs dont il est le support. Les dimensions matérielles du quartier leur permettent de se rappeler leurs moments passés. Elles leur servent à se rappeler et à se remémorer (Halbwachs, 1997). Dans le cas de Matta Sur, nous distinguons deux profils de personnes utilisant le quartier comme support à la remémoration.

Le premier concerne des personnes qui ne sont pas nées dans le quartier mais qui se servent de ses dimensions physiques et sociales pour se souvenir des lieux de leur enfance ou pour retrouver le genre de relations sociales qu'elles ont connu auparavant dans d'autres villes ou quartiers. C'est le cas par exemple d'un des dirigeants du Centro cultural Patrimonio Matta Sur qui a choisi de venir vivre à Matta Sur il y a neuf ans parce que le quartier lui rappelait celui où il a grandi. C'est le cas aussi de la directrice d'un autre centre culturel qui a décidé de s'installer à Matta Sur pour retrouver les mêmes relations de voisinage que là où elle est née, dans un village au nord de la capitale. Dans ces deux exemples, la relation affective se génère à partir d'objets, de personnes ou de lieux qui rappellent d'autres lieux qu'ils ont connus par le passé. La transformation de l'espace en support de souvenirs et de mémoires et donc de nostalgie passe ainsi par l'expérience individuelle vécue dans le passé.

Le second profil concerne les habitants qui ont vécu toute leur vie dans le même secteur et le même logement. C'est le cas de la majorité des participants. Le risque de voir leur maison détruite provoque chez eux de la peur et parfois de l'indignation qui les conduisent à agir pour faire obstacle à ces évolutions. Les promoteurs immobiliers viennent proposer parfois plusieurs fois par semaine l'achat de leur maison. Ces procédés sont souvent légaux mais ils utilisent aussi parfois la menace pour inciter les habitants à quitter et vendre leur logement. Cependant, pour ces derniers, leur habitat n'est pas seulement un lieu pour dormir mais plutôt un espace hérité de plusieurs générations et " habité " par les mémoires familiales. Ils sont d'abord attachés à leur maison et après par extension, ils éprouvent de l'affection pour le quartier. Aujourd'hui, l'argument principal des associations d'habitants est de présenter cet ensemble urbain comme un bien commun à ses habitants qu'il faut défendre pour les valeurs patrimoniales qu'ils lui associent. Cependant, le premier élément que les habitants veulent protéger est leur bien privé, leur propriété, autrement dit ce qu'ils ont hérité individuellement. L'espace patrimonial du quartier se construit ainsi par accumulation et partage de préoccupations individuelles vis-à-vis de patrimoines privés. Par ailleurs, à l'échelle du quartier, les habitants éprouvent aussi de l'attachement affectif pour les autres familles qu'ils connaissent depuis longtemps (ou même depuis toujours). Ils ont grandi ensemble et même si les conflits ne sont pas inexistants, ils se connaissent et se reconnaissent les uns et les autres.

L'affection que les habitants ont pour leur maison et plus généralement leur quartier est le point d'origine de la mobilisation. L'inquiétude et la peur de voir leur quartier se modifier par l'intervention de promoteurs et d'investisseurs obligent les habitants à exprimer collectivement et publiquement leur mal-être. Par leurs échanges, leurs discussions, leurs conversations et leurs projets en communs, ils partagent un même référent - le quartier auxquels ils associent le même type d'émotions et de significations : d'un côté, la fierté et 
l'affection basées sur les souvenirs et sur les relations de voisinage, de l'autre, l'indignation et la colère éprouvées par le manque de prise en compte des habitants par les institutions et le marché immobilier.

\section{Du partage d'émotions aux actions collectives}

La transition d'émotions intimes et privées à des émotions publiques et partagées est rendue possible dans le cadre de création "d'arènes publiques " (les groupes étudiés) de débats où les problèmes privés - la menace des promoteurs, le risque de construction de tours et plus largement la probabilité de voir le quartier changer - deviennent publics et politiques (Canteros, 2011). Par ailleurs, ces lieux de débats sont aussi et surtout des espaces d'échanges entre participants. Dans le cas des mobilisations d'habitants à Santiago et spécifiquement à Matta Sur, les associations et centres culturels remplissent cette fonction. Les ateliers pour élaborer le registre de photographies patrimoniales en sont une bonne illustration. Tandis que durant les premières classes théoriques le professeur ${ }^{3}$ insistait sur les dimensions intimes et privées du patrimoine, progressivement il s'est attaché à montrer comment le patrimoine pouvait être vu comme un bien commun. Les premiers exercices consistaient ainsi à prendre en photographie les éléments de notre vie quotidienne, nos espaces privés qui nous représentent le mieux. Le professeur voulait ainsi diffuser un message clair aux participants: "valoriser son patrimoine par la photographie, c'est se valoriser soi-même " (atelier du 07/04/15; traduction de l'auteur). Lors de l'atelier suivant, les participants devaient présenter aux autres leurs photographies et les expliquer. Cette première étape a ainsi servi aux participants à mieux se connaitre et à partager leurs expériences personnelles ou leurs souvenirs à partir de ce matériel visuel. A partir de ces premiers travaux et très rapidement, les conversations entre photographes amateurs se sont détachées de la sphère privée pour aborder des thématiques sociales liées à la protection du quartier et à ses modes de vie. Le partage d'expériences, d'opinions, de préoccupations et de craintes lors des réunions et activités a un effet amplificateur sur les émotions ressenties par les participants.

La transformation d'émotions individuelles en actions collectives se fonde sur des causes et des références communes identifiées grâce aux expériences vécues conjointement par les participants. Les émotions sont liées à des systèmes de valeurs qui justifient et animent l'action (Woods et al., 2012). Dans le cadre de la mobilisation patrimoniale, il est intéressant d'observer que les valeurs historiques ou mémorielles sont utilisées par les défenseurs du quartier avant tout comme argument auprès des institutions. Par exemple, aujourd'hui, le Comité de defensa del barrio Matta Sur demande la protection du quartier entier au nom de sa valeur patrimoniale qui serait constituée selon ses responsables par la combinaison et la complémentarité entre ses dimensions architecturales, mémorielles et sociales. Mais dans leur bulletin d'association, ils expliquent clairement qu'en 2007, ils se sont opposés au projet de corridor de bus Avenue Matta parce qu'il allait détruire l'espace vert au centre de l'avenue et parce que pour eux, ce lieu est chargé de souvenirs. De la même manière, les responsables du Centro cultural Patrimonio Matta Sur veulent revaloriser le patrimoine du quartier officiellement pour ses valeurs mémorielles et historiques mais lorsque nous observons de plus près leurs pratiques, il s'agit d'actions basées avant tout sur les souvenirs

\footnotetext{
${ }^{3}$ Il est photographe professionnel du musée historique national.
} 
et impressions des participants. Que ce soit les réunions pour la création d'un musée du quartier ou les ateliers de photographies patrimoniales, ils sont des moments de partage émotionnel entre participants, traduites par la suite en arguments et valeurs compréhensibles et accessibles aux personnes extérieures aux groupes.

Cette transposition d'émotions individuelles en émotions collectives se déroule à travers les conversations entre participants mais aussi grâce à l'utilisation des activités par les responsables des groupes comme dispositifs de sensibilisation qui font appel et animent des émotions latentes en chaque participant. Par exemple, le musée du quartier est élaboré avec l'aide d'habitants qui ont fait "don " d'objets, de photographies familiales ou de cartes postales historiques du quartier. Les propriétaires des biens ressentent de multiples émotions tels que l'amour, la joie, la tristesse lors de l'exposition. Mais les autres visiteurs aussi expriment ces mêmes émotions parce que les objets leur permettent de penser à leurs propres souvenirs auxquels ils attachent des valeurs et du sens. Nous pouvons ainsi dire que les émotions individuelles sont influencées par les cadres socio-culturels composés de valeurs, de significations et de sens partagés entre les habitants: les visiteurs et les propriétaires des objets ressentent et expriment les mêmes émotions parce qu'ils appartiennent aux même groupes sociaux et partagent les mêmes valeurs sociales.

Finalement, les émotions exprimées par les participants sont à la fois l'origine et le résultat des actions menées collectivement. D'un côté, les habitants se réunissent et s'unissent à partir de leur attachement affectif au quartier et à ses modes de vie. Ils cherchent à faire face ensemble à leur peur et leur crainte de voir ce qu'ils aiment disparaitre ou changer. D'un autre côté, une fois rassemblés, les discours et les activités proposées dans ces groupes engendrent des émotions collectives latentes qui ont davantage à voir avec la colère contre un système économique et politique et la fierté de faire partie d'un quartier patrimonial.

\section{Un groupe formé sur la base de normes émotionnelles}

Les participants considèrent qu'ils forment un « nous " à la fois pour les moments passés, les valeurs, les intérêts et les sensibilités qu'ils ont en lien avec le quartier. Le sentiment d'appartenance au groupe prend forme à la fois dans l'échange avec les autres membres et dans la confrontation à l'autre. Lors des réunions et des événements collectifs, les participants partagent leurs souvenirs du quartier et leurs idées sur ses évolutions futures. De plus, ils écoutent et soutiennent les discours des dirigeants qui défendent l'existence d'une lutte entre la "communauté " et les promoteurs immobiliers. Ils se sentent ainsi partie prenante d'un conflit les opposant à ceux qui ne penseraient pas de la manière l'évolution des quartiers et de la société en général.

Progressivement, dans ce "nous ", les membres se conforment à l'image collective qu'ils veulent donner au groupe à travers une autorégulation de leurs émotions et de leur comportement en relation aux autres participants. Les habitants qui veulent s'engager dans ces processus s'appliquent alors à respecter ces normes. Il serait par exemple impossible de participer et faire partie du groupe sans apprécier " prendre la once " (sorte de tea-time chilien) en fin de journée, sans parler de longues heures avec les voisins dans la rue ou les saluer en les croisant le matin ou le soir sur la place du quartier. Les participants doivent créer le " charisme du groupe " (Elias, Scotson, 1997) à partir de ces pratiques. Pour eux, les résidents des nouvelles tours ne partagent pas ces usages et coutumes. Perpétuer ces 
pratiques permet aux habitants mobilisés de se différencier des "nouveaux " habitants. II est ainsi possible d'assister lors des réunions à une exacerbation des réactions face à des images, à des pratiques ou à des habitudes qui correspondent au groupe, comme c'est le cas par exemple de l'atelier de photographies patrimoniales (Figure 6) où le professeur a l'habitude de montrer ses photographies personnelles.

\section{Figure 6 : Une des classes de l'atelier de photographies patrimoniales}

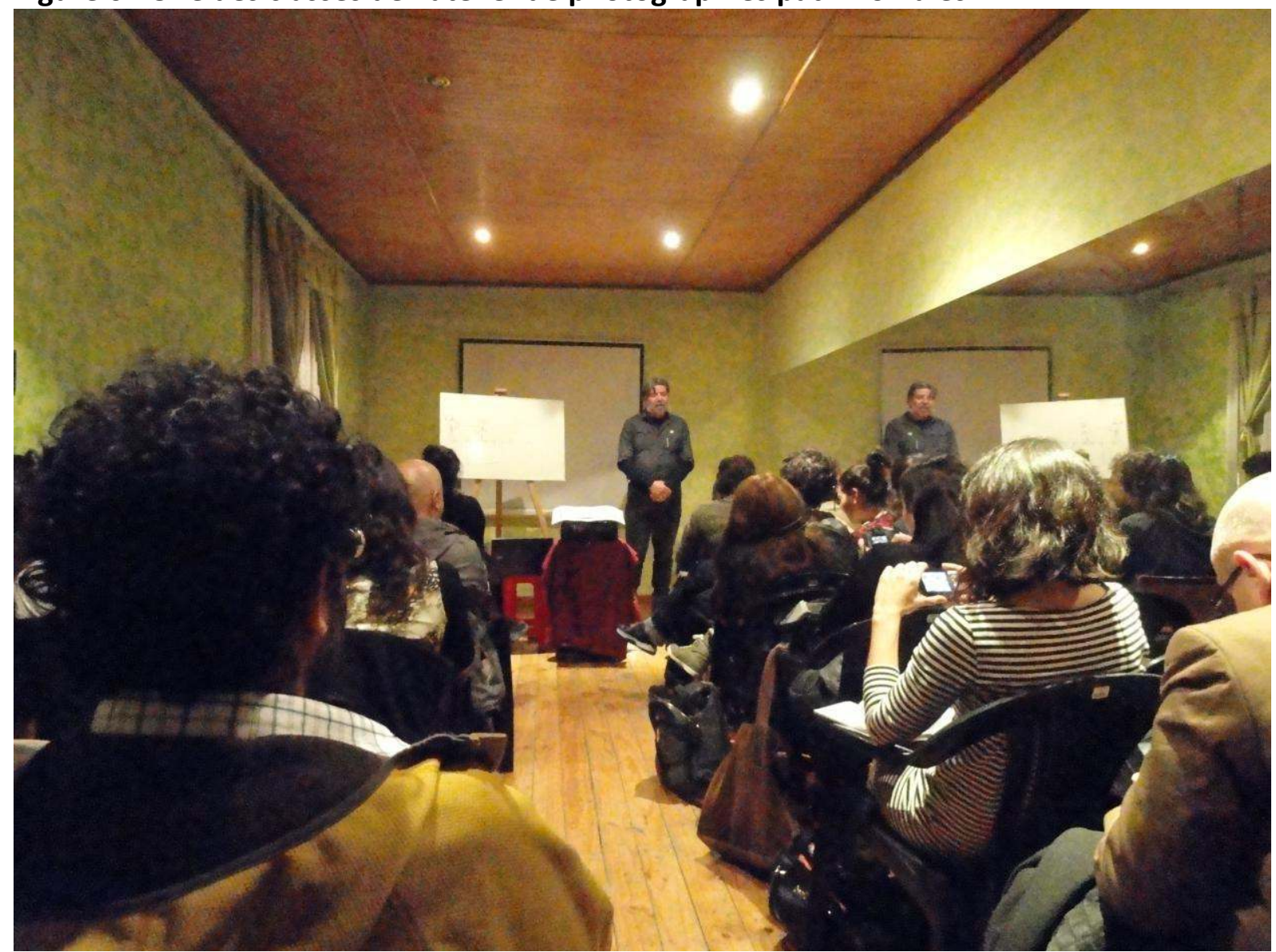

Cliché de l'auteur, juin 2015.

Face à des scènes quotidiennes - notamment les repas et en particulier la once - tous expriment de la joie, de l'envie et même de la jalousie montrant qu'ils veulent participer à cette scène. Les photographies de famille du professeur provoquent aussi de fortes réactions liées à de l'enthousiasme et de la tendresse. Autre thème abordé dans les classes : I'histoire nationale représentée par les photographies historiques du Santiago (et d'autres villes) de différentes époques. A chaque photographie, les personnes présentes aux classes devaient reconnaître le lieu et l'époque. Cependant, la plupart du temps, le lieu est aujourd'hui détruit ou totalement différent. Face à cela, les participants réagissent de manière similaire : "quel dommage! ", "qu'est-ce que c'était incroyable ! " ou " qu'est-ce que c'était beau! " (Traduction de l'auteur). Ces exemples présentent des types d'émotions permettant aux habitants de s'identifier, de se définir comme appartenant au groupe.

Par ailleurs, la structuration de ces groupes conduit à la formation d'une dichotomie forte entre "insiders" - les membres des anciennes familles du quartier qui participent aux activités patrimoniales - et " outsiders " - les nouveaux résidents qui se sentent souvent peu concernés par ces processus. Les dynamiques sociales observées ont pour conséquence 
l'exclusion des autres résidents et la constitution d'un "entre-soi ", c'est-à-dire le regroupement de personnes aux caractéristiques communes, sous-entendant l'exclusion, plus ou moins active et consciente, des autres au nom de leur sentiment de supériorité (Tissot, 2004). Les participants veulent revaloriser leur quartier en mettant à distance les autres habitants, en les dévalorisant pour ce qu'ils sont et pour ce qu'ils représentent : les nouveaux résidents non ancrés dans ce territoire et ne partageant pas leurs valeurs.

La distinction entre le groupe et les autres s'observe pour les immigrés péruviens, colombiens ou d'Amérique Centrale dont les communautés viennent trouver un lieu de vie dans le centre de Santiago. Cela peut se révéler dans un regard, une expression qui permet de comprendre la différence existante entre les membres du groupe et les autres. Cette distinction se vérifie aussi pour les nouveaux résidents de nationalité chilienne ne partageant pas les mêmes usages du quartier. L'exclusion du groupe est alors beaucoup plus subtile mais peut s'identifier à travers l'humour et les moqueries. Par exemple, une fois, lors d'une des réunions à laquelle nous avons assisté, une personne inconnue des autres participants a expliqué qu'elle vivait dans une des tours non loin du lieu où nous étions. La réaction d'un des animateurs était de dire avec humour et entre deux rires "dehors » à la personne visée. Bien entendu, la personne n'était pas exclue de la réunion mais ce type de comportement est révélateur des relations que ces groupes entretiennent avec les "autres". La revalorisation patrimoniale se base ainsi clairement sur ces relations complexes et ambiguës entre groupes établis et ceux que nous pourrions nommer " marginaux " (Elias, Scotson, 1997). Estimant connaitre parfaitement le quartier, ses mémoires et ses usages, ces groupes pensent détenir un pouvoir supérieur par rapport aux autres résidents.

\section{Conclusion}

Face aux changements socio-spatiaux engendrés par la construction de tours dans le centre de Santiago, des groupes d'habitants se mobilisent pour promouvoir un type de revalorisation des quartiers qui ne serait pas purement économique mais aussi patrimonial et culturel. Ce type de groupes se compose d'individus appartenant aux anciennes familles du quartier. Cependant, leur cohésion et leur entente mutuelle tendent à la formation d'un entre-soi, un "nous " sur la base d'émotions, d'expériences et de valeurs partagées lors des réunions et des activités qu'ils mènent dans le quartier. Le sentiment commun d'appartenir à une même communauté se construit d'un côté par les pratiques et les émotions collectives et de l'autre, par leur différentiation avec les nouveaux résidents.

Plusieurs émotions jouent des fonctions déterminées dans la mobilisation. Tout d'abord, la possible disparition du quartier et de ses modes de vie conduit certains habitants à éprouver de la peur et d'autres de la colère. Dans les deux cas, l'indignation provoquée par le sentiment d'injustice amène ces individus à s'opposer aux processus en cours. Ces émotions se forment parce qu'ils entretiennent des relations affectives fortes avec leur espace de vie à partir des mémoires et souvenirs qu'ils lui associent. Cette situation leur fait sentir de la nostalgie pour une période qu'ils ont connue et qu'ils aimeraient retrouver. Le maintien de la mobilisation est alors rendu possible grâce à l'existence d'autres émotions : d'un côté, la joie et la satisfaction de participer à une cause jugée juste et de l'autre, l'amour ou l'amitié éprouvé pour les autres habitants partageant les mêmes idées. Ces émotions conduisent 
néanmoins les participants à éprouver du mépris ou parfois de la haine pour les personnes à l'extérieur du groupe ou pour leurs opposants.

Par ailleurs, la mobilisation patrimoniale se déroule dans un rapport ambigu et permanent entre émotions individuelles senties et exprimées (consciemment ou inconsciemment) par chaque participant et construction/intégration d'émotions collectives en tant que normes sociales. D'un côté, les habitants se joignent au groupe à partir des relations affectives et intimes qu'ils entretiennent avec le quartier et ses habitants à partir de leurs souvenirs. De l'autre côté, ils s'entendent avec les autres participants car ils partagent les mêmes idées politiques mais aussi les mêmes mémoires, représentations et imaginaires sociaux qui se traduisent en émotions collectives préétablies et englobantes.

Pour conclure, nous pouvons dire que la mobilisation patrimoniale s'établit et se structure à partir d'une double dynamique émotionnelle. La première a pour fonction de former le groupe autour d'une référence commune: le quartier. Elle se base sur les émotions individuelles engendrées par les pratiques et les habitudes quotidiennes des participants. La seconde dynamique conduit et anime le groupe. Elle se fonde sur les discours des responsables des groupes d'habitants qui font référence à l'histoire sociale et aux mémoires collectives qui s'ancrent dans l'idée de " quartier ". II s'agit ainsi de dispositifs utilisés par les acteurs de la mobilisation pour éveiller des émotions collectives latentes chez les participants. Dans ce cadre, les habitants ne pouvant ou ne voulant pas intégrer les mêmes normes émotionnelles sont exclus de ces groupes.

\section{Bibliographie}

BOLTANSKI L., THEVENOT L. (1991), De la justification. L'économie des grandeurs, Paris, Gallimard, 483p.

CANTEROS E. (2011), « Las agrupaciones vecinales en defensa de los barrios. La construcción política desde lo local », Polis, 2011/10(28), pp. 85-99.

COLLINS, R. (2001). "Social movements and the focus of emotional attention ", in Goodwin, J., Jasper, J.M, Polleta, F. Passionate Politics: Emotions and social movements, Chicago, University of Chicago Press, pp. 27-44.

CONTRERAS Y. (2011), " La recuperación urbana y residencial del centro de Santiago: Nuevos habitantes, cambios socioespaciales significativos », EURE 2011/37(112), pp. 89-113.

DAMASIO, A. (1999). Le sentiment même de soi: Corps, émotions, conscience, Paris, Odile Jacob, 380p.

ELIAS N., SCOTSON J. L. (1997) [1966], Logiques de I'exclusion : Enquête sociologique au cœur des problèmes d'une communauté, Paris, Fayard, 278p.

FABRE, D. (Ed.) (2013). Emotions patrimoniales, Paris, Maison des sciences de l'homme, 408p.

FLAM, H., KING, D. (Eds.). (2005). Emotions and Social Movements, Oxford/New York, Routledge, 213p.

GOODWIN, J., JASPER J. M., POLLETA F. (2001), Passionate Politics: Emotions and social movements, Chicago, University of Chicago Press, 383p.

HALBWACHS M. (1997) [1950], La mémoire collective, Paris, Albin Michel, 295p.

HEINICH, N. (2012). "Les émotions patrimoniales: De l'affect à l'axiologie ", Social Anthropology/ Anthropologie sociale 2012/20(1), pp. 19-33. 
HIDALGO, R. (2010). "Los centros históricos y el desarrollo urbano inmobiliario: las contradicciones de un negocio exitoso en Santiago de Chile ", Scripta Nova, 2010/331(85), <http://www.ub.edu/geocrit/sn/sn-331/sn-331-85.htm>

ILLUSTRE MUNICIPALIDAD DE SANTIAGO. (2013). Memoria explicativa: proyecto de modificación al plan regulador de Santiago, sector $n^{\circ} 5$ Matta-Franklin y actualización del texto de la ordenanza local, Santiago, Chili, 128p.

JASPER J.M. (2012), "Las emociones y los movimientos sociales: veinte años de teoría e investigación ». Revista Latinoamericana de Estudios sobre Cuerpos, Emociones y Sociedad, 2012/10(4), pp. 48-68.

LE BRETON, D. (2004). Les passions ordinaires: Anthropologie des émotions, Paris, Payot, $346 \mathrm{p}$.

LUTZ, C. (1986) "Emotion, thought and estrangement: emotion as cultural category ", Cultural Anthropology 1986/3(1), pp. 287-309.

MCADAM D. (1999) [1982], Political Process and the Development of Black Insurgency (1930-1970), Chicago, The University of Chicago Press, 346p.

MCCARTHY J. D., ZALD M. (1977), « Resource, Mobilization and Social Movements: a Partial Theory ", American Journal of Sociology 1977/82, pp.1212-1241.

MELUCCI A. (1999), Acción colectiva, vida cotidiana y democracia, Mexico D.F, El colegio de Mexico, 260p.

OLSON M. (1992) [1966], La lógica de la acción colectiva, bienes públicos y la teoría de grupos, México D.F, Limusa- Noriega, 199p.

PODUJE, I. (2008). "Participación Ciudadana en Proyectos de Infraestructura y Planes Reguladores ", Serie de Temas de la Agenda Pública de la Vicerrectoría de Comunicaciones y Asuntos Públicos 2008/3(22).

PRATS, L. (2009). Antropología y Patrimonio, Barcelona, Ariel, 246p.

RAMÓN DE., A. (2000). Santiago de Chile (1541-1991): Historia de una sociedad urbana. Santiago de Chile, Editorial Sudamericana Chilena, 288p.

SABATINI, F., WORMALD, G. (2004). " La guerra de la basura de Santiago: desde el derecho a la vivienda al derecho a la ciudad ", EURE 2004/91(30), pp. 67-86.

SCHÜTZ, A. (1998). Éléments de sociologie phénoménologique, Paris, L'Harmattan, 156p.

TARROW, S. (1989). Democracy and Disorder: Protest and Politics in Italy 1965-1975, Oxford, Clarendon Press, 400p.

TILLY C. (1978). From Mobilization to Revolution, Addison-Wesley, Reading, 349p.

TISSOT, S. (2014). "Entre soi et les autres ", Actes de la recherche en Sciences sociales 2014/4(204), pp. 4-9.

TORRES A., HIDALGO, R. (2009). "Los peruanos en Santiago de Chile: transformaciones urbanas y percepción de los inmigrantes ॥, Polis 2009/22(8), pp. 307-326.

TRAïNI C. (Ed.). (2009). Emotions...Mobilisation!, Paris, Presses de Sciences Po, 300p.

TRAÏNI C. (Ed.). (2015). Emotions et expertises: Les modes de coordination des actions collectives, Rennes, Presses Universitaires de Rennes, 204p.

WOODS, M., ANDERSON, J., GUILBERT, S., WATKIN, S. (2012). "The country(side) is angry: emotion and explanation in protest mobilization ", Social and Cultural Geography 2012/13(6), pp. 567-585. 